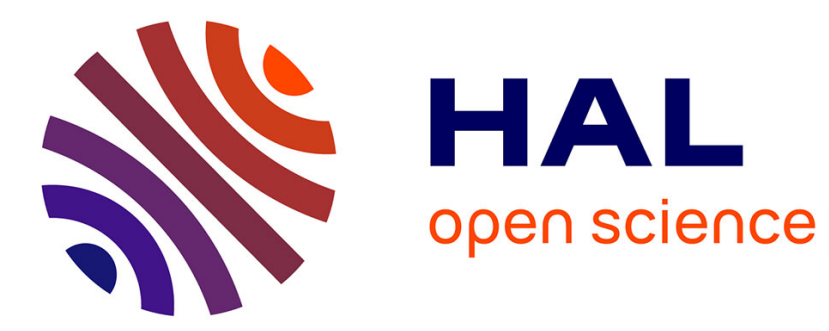

\title{
On the microscopic structure of liquid water
}

Aurélien Perera

\section{To cite this version:}

Aurélien Perera. On the microscopic structure of liquid water. Molecular Physics, 2011, pp.1. 10.1080/00268976.2011.617712 . hal-00739204

\section{HAL Id: hal-00739204 \\ https://hal.science/hal-00739204}

Submitted on 6 Oct 2012

HAL is a multi-disciplinary open access archive for the deposit and dissemination of scientific research documents, whether they are published or not. The documents may come from teaching and research institutions in France or abroad, or from public or private research centers.
L'archive ouverte pluridisciplinaire HAL, est destinée au dépôt et à la diffusion de documents scientifiques de niveau recherche, publiés ou non, émanant des établissements d'enseignement et de recherche français ou étrangers, des laboratoires publics ou privés. 


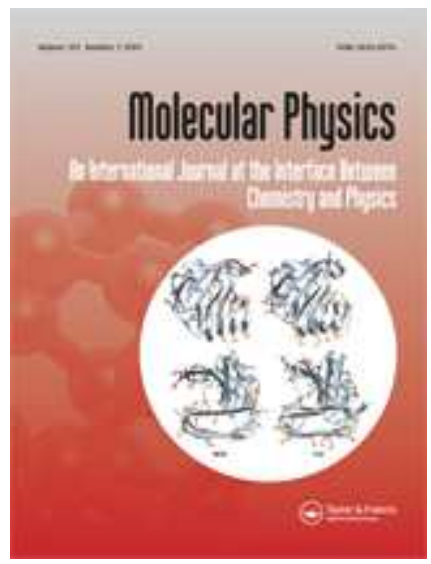

\section{On the microscopic structure of liquid water}

\begin{tabular}{|r|l|}
\hline Journal: & Molecular Physics \\
\hline Manuscript ID: & TMPH-2011-0253.R2 \\
\hline Manuscript Type: & Full Paper \\
\hline $\begin{array}{r}\text { Author: } \\
\text { Keywords: }\end{array}$ & 19-Aug-2011 \\
\hline $\begin{array}{r}\text { Complete List of Authors: liquid, simulations, structure } \\
\text { Therorique de la Matière Condensée }\end{array}$ & $\begin{array}{r}\text { Pera, Aurélien; Université Pierre et Marie Curie, Lab Physique } \\
\hline\end{array}$ \\
\hline $\begin{array}{l}\text { Note: The following files were submitted by the author for peer review, but cannot be converted } \\
\text { to PDF. You must view these files (e.g. movies) online. }\end{array}$ \\
\hline $\begin{array}{l}\text { figures.zip } \\
\text { waterStruct.tex }\end{array}$
\end{tabular}

\section{SCHOLARONE \\ Manuscripts}


(3) "The author is not expert in the experimental uncertainties of neutron scattering data due to inelastic scattering in the region of $3 A-1$. Please remove sentence:

"A closer look at the neutron SF reported in Ref 27 nevertheless reveals a small hump at $k 3 \AA-1, a$ weak sign of the H-bond ordering."

The incriminated sentence have been now removed.

(4) "I do not agree with the summary of Guillot in 2002 (a reference 10 years out of date to current water models)

"It is well acknowleged that polarisable force field model for water are generall speaking not as good as non-polarisable force fields. "

That work showed that some properties are better and some worse using polarization (again I emphasize a study done 10 years ago!) and that fixed charge FFs (10 years ago) were the same, not better or worse. That sentence should be deleted."

Even if the Guillot paper is now 10 years old, it has a very high citation index and seems to be a reference for many water experts in the community, from what I have personally gathered. Many things he says about polarisable force fields are still true I believe. Nevertheless, I have complied with the referee asking and removed this sentence from the manuscript.

(5) "Reference on page 2 marked as "?""

I thank the Referee for mentioning this glitch to me. I have corrected it now. The missing Reference is to Guillot [11].

(6) "The following sentence is very unclear and needs to be restated so that readers can follow the authors's point: 
"One suspects that this long range "ideality" is only apparent, and it hides canceling effects, but which?" "

This sentence means to say that, if long range correlations beyond 10A are zero, then the effective mean force potential is also zero in this range, which also means that particles do not interact and therefore appear as "ideal" particles in this very range. However, this is certainly not true, and some cancellation effects must be hiding behind this feature. I have now modified the first part of this sentence according to what written above.

(1) I find that the Referee is complaining now about material that were already present in the first version of the manuscript, and about which he did not raise any comments in the first refereeing part. Anyhow, I will break up the various points to ease the answers.

"I find the introduction confusing as to what point the author is trying to make. It seems to involve a mish mash of statements about local tetrahedral structure vs. mixture models. The author brings up references to changing structure with temperature when his study is at room temperature. He references the Huang et al study as if underlying heterogeneous structure and mixture models have found experimental verification. "

The only changes that were brought to the Introduction were based on the previous comments of the Referee about the usefulness of the reference to Huang et al. [15-16] precisely. I could not find in the text the statement(s) where I say that their claims have found experimental verifications. As to the temperature dependence, it is to mention the evolution of the tetrahedral structure.

The purpose of the Introduction, as I wrote it initially, is very clear to me. It is all about to briefly describe the state of matters about the tetrahedrality of water and the various hypothesis about the underlying statistical structure of the liquid. I found this useful before I bring up the main finding of this paper which is the 3PS. The description of the historical background may not be perfect in it present form, but it does gives an overall idea of the past and current state of knowledge, in my opinion.

"I also don't believe that the water field is so naive as to base their analysis on "instantaneous snapshots" and that we are all unaware of statistical mechanics and ensemble averaging."

The statements that the Referee criticise, were already present in the initial version of the manuscript. The point is that the immediate tetrahedral structure around a single water is often imagined (and pictured in many articles and presentations!) as a that from the crystal. I think that such representations are directly inspired by snapshot visualisations. I have altered some statements in this paragraph to appear less "offending" as the Referee seems to suggest. Also, the paragraph serves initially to introduce the pair correlation as a proper observable, which then leads naturally to investigate the 3PS. In that sense, I think that the whole paragraph is quite coherent.

"In essence the author wants to make a rather straightforward point: that tetrahedral hydrogenbonding interactions and packing effects compete so that their long-range *individual* correlations cancel each other beyond 3 molecular diameters. And furthermore this effect need not be explained as a mixture model of a LJ liquid and ice, but instead is consistent with homegeneous fluctuations in the bulk liquid."

I am afraid I do not understand what the Referee is suggesting, at least not clearly enough to take the risk of altering the manuscript subsequently. Moreover, I do say in the manuscript some of the statements that the Referee suggests, as for example that the mixture model is not correct (in the Conclusion for example). The effective cancellation of correlations is also many times mentioned in 
the manuscript.

"Certainly the introduction could be better written to state these points. In fact the conclusion section is more clear on these points, and maybe that discussion could be moved to the introduction."

I thank the Referee for his positive statements on the Conclusion. I wish the Referee had exactly said which points he wanted me to move to the Introduction. I personally find that the topics in the Introduction are very different than in the Conclusion. There, I was setting up the scene to introduce the 3PS and subsequent facts, while in the Conclusion I dwell on the consequences of the 3PS finding. In the doubt, I have preferred not to alter these two parts. 


\title{
On the microscopic structure of liquid water
}

\author{
Aurélien Perera \\ August 19, 2011 \\ Laboratoire de Physique Théorique de la Matiére Condensée (UMR CNRS \\ 1600), Université Pierre et Marie Curie, 4 Place Jussieu, F75252, Paris cedex \\ 05, France.

\begin{abstract}
The radial distribution function of liquid water, as obtained by the computer simulations of several classical models of water, is reexamined herein and shown to displays two intriguing features. These consist in a compact "three-peaks structure" over three molecular diameters, which is followed by an apparent loss of the packing correlations beyond $R_{c} \approx 9 \AA$. This is in contrast with simple liquids for which the correlations decay continuously with distance. This structure is reproduced for many widely used classical force fields models of water and by scattering experiments as well. It is also preserved in aqueous binary mixtures of organic solvents, even up to equimolar mixture in some cases. The analysis of the structure factor highlights the role played by the competition between packing effect and the Hydrogen bonding interactions. This analysis, in terms to competition of two length scales, is also supported by a simple core-soft model, that reproduces the structural features outlined above.
\end{abstract}

\section{Introduction}

Water is certainly the natural substance that has the most known anomalous properties. These properties arise ultimately from the very specificity of the pair interaction between water molecules, namely the hydrogen bond. The resulting local tetrahedral structure of liquid water has been the subject of many tentative descriptions over the past decades, among which the two-waters mixture (or iceberg) model ${ }^{1-3}$ and the hydrogen bond percolation model, ${ }^{4}$ as well a combination of both. ${ }^{5}$ Although such structural models provide an intuitive picture of the cooperative extension of the local Hydrogen 
bonded (H-bond) tetrahedral structure ${ }^{6}$ the various experimental investigations, either by structural analysis ${ }^{7,8}$ or by various spectroscopic techniques, ${ }^{9}$ are not directly supportive of such pictures, although never clearly stated. For example, it is not possible to observe two different motions of water at the very short times, ${ }^{9}$ which would confirm that two types of water environments exist. On the other hand, neutron experimental ${ }^{10}$ studies of the temperature dependence of the position of the first peak of the structure factor, indicate that the local environment of a single molecule goes from tetrahedral-like at low temperatures to disordered at higher one. The numerous interaction models of water ${ }^{11}$ and their computer simulations also do not provide direct conclusive evidence to support these ideas about the structure of liquid water. More recently introduced water-like models, such as the Jagla model, ${ }^{12-14}$ for example, indicate that the corresponding liquid might coexist under two different forms, although at temperatures corresponding to the supercooled regime. Similar discussions on the possible underlying heterogeneous structure of liquid water has been conducted even very recently. ${ }^{15-18}$

The intuitive pictures that guide the ideas about the local structure of a liquid are often based on an instantaneous snapshot-like visions. This is particularly true for water and the ice-like tetrahedral disposition of the water molecules around a single central molecule. In Statistical Mechanics however, such snapshot corresponds to one micro-state, whereas any measure of a system at equilibrium should correspond to an average over all possible such micro-states. The question is then to figure out how such successive averages keep or destroy the picture made out of one snapshot. Such question makes no sense for an ordinary liquid that is essentially disordered, but in the case of water, which is strongly ordered at molecular level through the H-bond network, such question does not lead to a trivial answer. Therefore, one may ask if the peculiar properties of liquid water can be seen directly on the averaged structure, which statistical representation is the pair correlation function. ${ }^{19}$ We wish here to emphasize that this correlation function is an observable of the statistical description of any liquid. This function has been reported many times for the case of liquid water, but only the molecular structure corresponding to the first two peaks is usually discussed. ${ }^{7,8}$ The peculiar ordering in liquid water may need a detailed investigation of what happens at larger molecular separations. Such a study, conducted here for several popular water models, and under various system sizes, reveals that the short and long range ordering of liquid water is indeed very different than that of any simple liquid. We analyse here how these findings are compatible or not with previous structural models, and if newer pictures can be formulated. 


\section{Computer simulations and results}

Herein, we focus on three popular and robust interaction models of water, namely the SPC/E ${ }^{20}$ the TIP $4 \mathrm{P}^{21}$ and the TIP $5 \mathrm{P}^{22}$ models. All these models consists of a single Lennard-Jones site with several embedded point charges corresponding to that of the oxygen and the two hydrogen atoms. Constant NPT Molecular Dynamics simulations have been conducted for the ambient conditions, temperature of $25^{\circ} \mathrm{C}$ and pressure of $1 \mathrm{~atm}$. Berendsen thermostat and barostat were used, with relaxation times of $0.1 \mathrm{ps}$ and $0.5 \mathrm{ps}$, respectively. The equations of motion were integrated with a time step of $2 \mathrm{fs}$, which is generically used in modern simulations of water and aqueous mixtures. Earlier simulations with a time step of $0.5 \mathrm{fs}$ did not give appreciably different results ad far as static properties are concerned, such as in the present work. The Lennard-Jones (LJ) interactions were truncated at one third of box size and Ewald summation was used for the Coulomb interactions. Ewald summation is usually slower than reaction field techniques, but it is a rigorous account of the Coulomb long range interactions in periodic system, and does not suffer from cutoff artifacts or the need to impose the bulk dielectric constant. For the computation of the sum in the reciprocal space, 10 reciprocal space vectors in each direction were used, with a spherical cutoff for the reciprocal space sum of $n_{x}^{2}+n_{y}^{2}+n_{z}^{2}<105$. The width of the screening Gaussian was set to $0.36 \AA$. The values In order to probe the long range part of the pair structure, we have used a large number of particles, namely $\mathrm{N}=2048$ water molecules. In order to test any possible system size dependence to the feature reported here, several system sizes were investigated, ranging from $N=864$ to $N=10976$ water molecules. It is important to note that the interaction and force cutoff scales with the system size since the LJ interactions are truncated at half-box length. For each system, the radial distribution function (RDF) was sampled every 50fs, between 1000 to 2000 times. These statistics ensure smooth enough RDF that can provide noiseless structure factors upon direct Fourier transformation. The generic features of the force field models, as well as the differences between them, are well known, ${ }^{8}$ and do not need to be repeated here.

\subsection{The structure of liquid water}

In order to fix the general idea about the structure of a simple disordered liquid, we report in Fig.1 the radial distribution functions $g(r)$ for a typical Lennard-Jones liquid, with a molecular diameter similar to that of water $\sigma_{W}=3.165 \AA$ and energy parameter $\epsilon / k_{B}=100 \mathrm{~K}$. Two densities have been considered, one with box size is $L=40 \AA$ and $N=2048$, which corresponds 
the reduced number density of water $\rho^{*}=\left(N / L^{3}\right) \sigma_{W}^{3} \approx 1$, under ambient conditions, and the other corresponds to a lower density of $\rho^{*}=0.73$, with $L=44.64$ Âwith the same number of particles. This latter density would correspond to that evaluated for a smaller water-water "contact diameter" $\sigma_{\text {eff }} \approx 2.84$, corresponding to the first peak of the experimental RDF of water. The reason for considering this lower density is to follow the reasoning found in the literature that water would appear as less dense because water molecules could come into contact at a smaller distance -that of the hydrogen bonding distance- than the actual molecular diameter $\sigma_{W}$. Fig.1 shows that the molecular packing typical of liquid like order is seen to extends to many molecular diameters, in both cases. This is particularly visible in the inset, when showing the integrand $r^{2} h(r)$ of the isothermal compressibility ${ }^{19}$ $(\partial \rho / \partial \beta P)_{T}=\int d \vec{r} h(r)$, where $h(r)=g(r)-1, \rho$ is the number density, $P$ the pressure and $\beta=1 / k_{B} T$ the Boltzmann factor. The remarkable point is that the envelope of the decay of the oscillations is a smoothly decaying function of the distance.

Turning now to water, the radial distribution functions between the oxygen atoms $g_{O O}(r)$ are shown in Fig.2, for all the models investigated here. There are well known differences between this RDF and that of a comparative simple liquid: ${ }^{8}$ the first peak is narrower and shifted inward, because of the strong directional $\mathrm{H}$-bond interaction. As a result, the coordination number in the first shell is about 4 instead of 12 as for an ordinary LJ liquid. Here, we wish to examine the structure beyond the first two peaks. The first important observation is that the oscillatory structure, which witnesses the molecular packing of neighbouring shells, is distinctly seen to extend over three molecular shells: this is what we call the 3-peaks structure (3PS) of water. The second important point is that the oscillatory structure corresponding to the molecular packing appears to be strongly diminished and noisy beyond $r \approx 9-10$. In order to see this feature more clearly, we show in the upper inset the same integrand as in Fig. $1 r^{2}\left(g_{O O}(r)-1\right)$ for the $\mathrm{SPC} / \mathrm{E}$ model and $\mathrm{N}=10976$. This magnified scale shows quite well the striking diminution of structure beyond $R_{c} \approx 9-10 \AA$, which is close to 3 molecular diameters. Since the $r^{2}$ term should amplify the oscillations due to packing (as in Fig.1), their obvious absence beyond this cutoff indicates that the packing correlations beyond $R_{c}$ are of a different nature than those for smaller distances. When compared to corresponding features of ordinary liquid in Fig.1, the contrast is striking, and reveals that water has very distinct features extending much further than immediate neighbourhood of a single molecule. Fig. 3 shows that similar structural features occur also for the oxygen-hydrogen $(\mathrm{OH})$ and the hydrogen-hydrogen $(\mathrm{HH})$ correlations. In particular, the diminution of the structure, almost like a random noise be- 
haviour of these functions seems to settle rather at $R_{c}^{\prime} \geq 12 \AA$, which is one molecular layer beyond that for $g_{O O}(r)$, as can be observed from the $r^{2} h(r)$ displays.

Fig. 2 and Fig.3 are the principal result of this report. We wish to emphasize that the 3 site-site distribution functions provide indirectly a description of the angular correlations as well, since these are embedded inside these site-site functions from their statistical definition. ${ }^{19}$ So, the fact that the features reported here show up in all three site-site function indicates that orientational contributions play an important role. The details of structure shown here is a very intriguing result, and to our knowledge has never been reported before. The observation of this structure demands large N simulations. Similar studies for different models and different system sizes, ranging from $\mathrm{N}=864$ to $\mathrm{N}=10976$, give the same results, as shown in Fig.4. We note again that increasing the system size and truncating the interaction at halfbox size remove all doubts that such features could arise from an artifact due to computational procedures. We also note that the TIPn models, while differing slightly at first and second shell, give strikingly similar results beyond these two shells. This indicates that the tetrahedral structure of all these models is more important than the small details by which these various force fields might differ. A closer look at the noisy structure beyond the 3PS reveals some remnant substructure that depends on models and simulation conditions. The TIPnP model simulations for $\mathrm{N}=2048$ show this structure more pronounced that the corresponding SPC/E model simulations. However, this smaller structure depends also on the system size, and is more pronounced for smaller $\mathrm{N}=964 \mathrm{SPC} / \mathrm{E}$ system than for the $\mathrm{N}=10976$ same system displayed in Fig.2. Resolving the issue of this smaller structure would require excessively accurate simulations on very large systems. The main point that we retain here is that the envelope of the oscillations drops abruptly after the 3PS, which is a behaviour very different from that of an ordinary liquid (Fig.1).

At this stage, a comparison with experiments is in order. This is shown in Fig.2, with scattering data from two references, Ref. ${ }^{7}$ and Ref., ${ }^{8}$ and it reveals two things. Firstly, the 3PS is also reproduced by the two sets of experiments. Secondly, however, currently available experimental data do not extend far enough to allow a proper evidence for the strong damping of correlations beyond $R_{c}$. Those extracted from $\operatorname{Ref}^{8}$ show clearly the 3peaks structure, on all three panels, but the data does not extend beyond $r=10 \AA$. An additional problem concerning this last feature arises. Since the $\mathrm{RDF}$ is extracted from the scattering data through the obtention of the structure factor, it involves several numerical operations, and in particular a very accurate estimate of the of the region of k-vectors between the first 
peak and $\mathrm{k}=0$ is required, in order to get an unambiguous long range part of the RDF. Since the corresponding radial distribution functions, shown in very many publications, are always shown for $r<10 \AA$, one may ask what is the accuracy of the estimate beyond this range. I hope that this report stimulates more accurate and detailed picture of the correlations beyond $10 \AA$. Similar remarks equally holds for quantum simulations of water, for which the small box sizes, imposed by the steep computational requirements, are not large enough to see the 3PS of water, and even less the abrupt end of the packing structure. We conjecture here that accurate experiments and quantum simulations should equally show this peculiar structure, for physical reasons that we discuss in the next section.

A first interpretation of these two features could be as follows: water molecules sense the packing effects only over a correlation shell of 3 molecular layers. Beyond this shell the bulk liquid is sensed as if it was a ideal media without packing effects whatsoever. This is very surprising, since ambient water is very dense, with reduced density of $\rho^{*}=1$, as can be deduced from the mass density $\rho_{m} \approx 1 \mathrm{~kg} / \mathrm{l}$. For comparison, the triple point density of an ordinary liquid is $\rho^{*}=0.8$. The long range behaviour observed in Fig. 1 for a LJ liquid under similar conditions enforces the puzzling behaviour observed for water. Since these long range correlations seems suppressed, one can infer that the mean force potential in this range is zero, and therefore that the particles do not interact with themselves in this range. One suspects that this long range "ideality" is only apparent, and it hides canceling effects, but which?

\subsection{A Core softened model of water}

In order to provide a qualitative explanation for the features observed, we consider a core-softened model of water that we have studied recently. ${ }^{23}$ Like many such models for water, ${ }^{12-14}$ the idea behind such models is to mimic the low coordination number of water by depleting the usual number of first neighbour of an ordinary liquid. This is usually obtained by adding a soft repulsive core. In our model, this core is modeled by a Gaussian interaction added to a soft sphere fluid with a repulsive $1 / r^{12}$ interaction. The LJ attractive interaction is intentionally removed to focus solely on entropic effects of local organization. The interaction used here is

$$
v(r)=4 \epsilon\left(\sigma_{W} / r\right)^{12}+\epsilon_{G} \exp \left(-\alpha_{G}\left(r-r_{G}\right)^{2}\right.
$$

with $\epsilon_{G} / \epsilon=30, \alpha_{G}=3.7 / \sigma_{W}^{2}$ and $r_{G}=0.85 \sigma_{W}$. Such a model is meant to mimic the two distances that are present in real water, namely the molecular 
diameter $\sigma_{W}$ and the shorter hydrogen bonding distance $\approx 2.5 \AA$. However, in the present model, these 2 distances are inverted: the Gaussian distance would correspond to the water diameter, and the inner core would be the $\mathrm{H}$-bonding distance. Despite this apparent caveat, this model has surprising structural analogies with water. The resulting RDF is shown in Fig.5, in addition to the $\mathrm{RDF}$ of the $1 / r^{12}$ soft core alone shown in dashed line. Both $\mathrm{RDF}$ are obtained from an NVT Monte Carlo simulation with $\mathrm{N}=4000$ particles. $^{23}$ Whereas the RDF of the soft-sphere fluid shows a concentric shell packing typical of simple liquid, that extends very far, the addition of the depleting Gaussian interaction is seen to have two effects. Firstly, because we have added a supplementary repulsion, we would expect the oscillations in the correlation function to move away from the main peak instead of closer to it -as it is observed here. This feature indicates that a specific structural reorganisation has occurred, which seems to bring particles closer than expected, as a result of the competition between the dense packing and the additional repulsion. Secondly, the correlations beyond $3 \sigma_{W}$ are strongly damped, which appears then to be the result of this enhanced short range structure. The resulting correlation function is clearly seen to be very much water-like, with the two features reported above. The diminutions of correlations beyond $3 \sigma_{W}$ is not as pronounced as for water. Nevertheless, it is striking that an isotropic interaction model should capture most of the water structure. The reorganization induced by the Gaussian repulsion is then to enhance correlations at short range, around a shell of radii $3 \sigma_{W}$, and the damping of correlation beyond seems the result of this local constriction. It therefore indicates that correlation beyond $3 \sigma_{W}$ are damped as the result of long range competing effects.

This study shows that a simple two-core model is able to reproduce features similar to real water, within the statistical observable of the structure, namely the radial distribution function. Beyond the apparent frustrating simplicity of the model, it reveals the underlying features that may help understand the special structure of real water, namely the presence of 2 contact distances.

\subsection{Influence of solutes}

What happens to this remarkable structure when water is mixed with an organic solute? In Fig. 6 the water-water RDF obtained from simulations RDFs is shown for three binary aqueous mixtures, for low (20\%) and equimolar $(50 \%)$ solute contents.

The solutes are acetone (OPLS model ${ }^{25}$ ), methanol (OPLS model ${ }^{24}$ ) and formamide (Cordeiro model ${ }^{14}$ ), which cover typical polar solutes with very 
different hydrogen bonding ability to water. Acetone is an H-bond acceptor with a large hydrophobic group of two methyl groups, methanol is the smallest alcohol and amphiphilic molecule, with both donor and acceptor abilities, and formamide is a typical polar solvent with no hydrophobic groups. All three solute mix with water in all proportions. Different water models are used, to show the universality of the features. The TIP4P model is mixed with acetone, and the SPC/E water model is mixed with the two other solutes. The structural results depend on the choices for different models of solute or water force fields, as was illustrated in Ref., ${ }^{26}$ but the main features that we want to focus on herein are invariant. All simulation have been done with $\mathrm{N}=2048$ particles with conditions similar to the neat water. The most striking feature in Fig.5 is that the 3PS of water is preserved for all these three mixtures and for the different solute concentrations. There is some loss of the 3PS in the case of the equimolar aqueous-formamide mixture, which is presumably due to formamide molecules being able to mix well with water because of the absence of hydrophobic groups and 3-fold H-bonding ability. One should keep in mind that all these results involve models. However, the similarities indicates a feature that should be a property of the real systems. The persistence of the 3 peak structure in mixtures indicates that water preserves its local H-bond structure, even in presence of large amounts of solutes. This seems to indicate that water molecules form coherent local structure between themselves, that are robust and persistent enough to survive in presence of a relatively dense solute environment.

\subsection{Structure factor}

Long range correlations are best observed in the reciprocal space, thus the structure factor $(\mathrm{SF})$ is a key point in the investigation. Indeed, this quantity is obtained directly from various neutron or X-ray scattering experiments. These can be compared with the SFs calculated from the RDF obtained in our simulations. The SF is simply the Fourier transform of the RDF $S(k)=\int d \vec{r} \exp (i \vec{k} \cdot \vec{r})(g(r)-1)$.

First of all, in order to have an idea of what to expect, we show in Fig. 7 the SFs of the core-softened model studied above. The structural changes from the SF of the soft $1 / r^{12}$ interaction to the SF when the Gaussian interaction is turned on, are twofold. First, the global oscillatory structure is seen to be shifted outward, which confirms again that the resulting packing structure is constricted, as observed in Fig.5. Second, the first peak is broadened and split in two. This can be almost considered as a two-peak structure, as if the main peak was shifted outwards and a smaller "pre-peak" feature had emerged. However, the interpretation in favour of the pre-peak is not correct, 
because, as was shown in Refs, ${ }^{34,35}$ there is no distinct cluster structure that could be associated with such a pre-peak, in contrast to what is observed for alcohols. The split peak structure is then a direct consequence of a dual structural correlations observed in the RDF, namely both the 3PS and the loss of correlations at long range.

Returning now to the SF of water, we plot in the top panel of Fig. 8 the $\mathrm{OO}, \mathrm{OH}$ and $\mathrm{HH}$ partial SFs for the $\mathrm{SPC} / \mathrm{E}$ model, together with the $\mathrm{OO}$ experimental SF, as measured by X-ray diffraction. ${ }^{8}$ The two compare quite well and despite some minor glitches, the principal features are reproduced. Other water force field models compare equally well and are not shown. The most important feature is the first split-peak, which is unexpected for a single component liquid.

In the following, we focus on the split peak feature. In simple liquids, as shown in dashed lines in Fig.7, this main peak is positioned at a k-vector $k \approx 2 \pi / \sigma$, where $\sigma$ is the atomic diameter. ${ }^{19}$ A pre-peak can be observed in systems such as micro-emulsions, ${ }^{33}$ which corresponds to the characteristic mean size of the micelles or any other structure that can form, such as bicontinuous domains or lamellae. ${ }^{33}$ In associated liquids, such as alcohols, a pre-peak can appear that corresponds to the formation of clusters. ${ }^{34,35}$ In view of these facts, the interpretation of the split-peak of water is very peculiar. The inner part of this peak is positioned at $k \approx 2 \AA^{-1}$, corresponding to $\sigma_{w} \approx 3.15 \AA$, the diameter of the water molecule. The position of the taller part of the main peak is at $k \approx 3 \AA^{-1}$, corresponding to the $\mathrm{OH}$ contact distance, estimated at $\sigma_{O H} \approx \sigma_{w} / 2$ from Fig.3. It is then remarkable that the inner peak is the main structural peak, due to molecular packing, while the taller second peak(or "outer-peak") is due to the H-bonding correlations. Since both are part of the main peak of the SF, it means that packing and Hbonding structural arrangement must have distinct statistical contributions, and form the basic structure of the correlations in the entire liquid. With this respect, it is quite interesting that the neutron scattering, which sees only the structural distribution of neutrons in the liquid, leads to a SF with a single peak, ${ }^{27}$ precisely centered at the packing structure at $k \approx 2 \AA^{-1}$, while the X-ray scattering, which sees the electronic structure, is able to report the splitting of the peak due to the H-bonding structure at a larger k-vector.

Following the analysis in amorphous ice, ${ }^{32}$ these structural features can be further clarified by analyzing the underlying Bathia-Thornton SFs, ${ }^{28}$ which would correspond to the density-density (NN) and charge-charge (CC) correlations, as if water was an atomic mixture of oxygen and hydrogen atoms. These structure factors are defined $\mathrm{as}^{28}$

$$
S_{N N}(k)=x_{O}^{2} S_{O O}(k)+x_{H}^{2} S_{H H}(k)+2 x_{O} x_{H} S_{O H}(k)
$$


in terms of the previous SFs and the mole fractions of the oxygen and hydrogen atoms, $x_{O}=1 / 3$ and $x_{H}=2 / 3$, respectively. Note that in the original definition the CC structure factor stands for concentration-concentration and measures the fluctuations in the repartition of the two species, which are here $\mathrm{H}$ and $\mathrm{O}$, hence the renaming into charge-charge. These new SFs are represented at the lower panel of Fig.8. They are nearly structureless since the molecules are rigid, hence there is no fluctuation in the atomic positions. However, it is clearly seen that the split-peak structure has been now distributed between $S_{N N}$ and $S_{C C}$. The main peak of the density-density SF $S_{N N}(k)$ is exactly at the pre-peak corresponding to the packing structure $k \approx 2 \AA^{-1}$, while the charge-charge peak for $S_{C C}(k)$ is positioned exactly at $k \approx 3 \AA^{-1}$.

These facts confirm that the features of the split peak are governed by the dual competition effects of packing and H-bond correlation effects. The apparent loss of packing correlations seen at long range is therefore the result of a compensation effect to enhanced $\mathrm{H}$-bond $\mathrm{OH}$ correlations. The simple isotropic model confirms that this effect is more of an entropic origin -due to local reorganizations - than energetical, in the sense of $\mathrm{H}$-bond energetics. Thus water is very strongly ordered at all scales.

\section{Outlook and conclusion}

The picture that finally emerges from all these results, is that water-water correlations are dominated by $\mathrm{H}$-bonding and packing correlations that appear to cooperate constructively at short distance, within a radius of $R_{c} \approx$ $3 \sigma_{W}$, and to cooperate destructively beyond, giving rise to the strong diminution of the correlations observed in all models. In view of the high packing density of water, this observation seems to suggest that the H-bonding correlations must be very important beyond $R_{c}$, since they nearly obliterate the natural packing correlations that should exist in this region, just like for the LJ liquid in Fig.1. Therefore, the apparent absence of correlations seems to hide some "mixture" of correlations that extend quite far in the bulk. At the same time, however, we do not see any sign that such correlations are accompanied by long range density correlations, as in a liquid close to the spinodal for example. These correlations appear therefore as structural -as part of a special local structure, and not induced by density fluctuation. With that respect, small angle X-ray scattering has revealed ${ }^{29}$ weak enhancement of the 
structure factor at $\mathrm{k}=0$, even at room temperature quite far from from any critical phase transition, which may provide some indirect support for special type of correlations that are not critical fluctuations as such. ${ }^{17,18}$ The field theoretic approach formulated by Tanaka, ${ }^{30}$ based on the idea of the competition of a density field and an H-bond ordering field, offers some support to the BT analysis of the structure factor.

In this study, we have have examined models without polarisation. One may ask if polarisation effects would alter the picture of the 3PS. Since the origin of the 3PS is the existence of 2 cores, that of the molecular diameter and the Hbonding distance, it is hard to see how polarisation effects can suppress this duality. They would rather change slightly their magnitude. In other words, we expects that more realistic force field models of water will reproduce the 3PS. One way to check this conjecture would be to obtain accurate scattering data for the correlations beyond the $10 \AA$ Arange in which they actually appear to be confined.

From methodological point of view, the analysis of the structure of water based on a statistically and computationally accessible quantity, namely the pair density correlation function, is probably more precise than considerations based on the analysis of thermodynamical quantities. As stated in the Introduction, it is a statistical observable of both experimental and computational studies. The result of the analysis provided here is not directly supportive of the two models mentioned in the Introduction, the iceberg model or percolated liquid model. The fact that our previous direct cluster analysis of water did not reveal the existence of distinct aggregates, such as those which were found in simple alcohols, ${ }^{34,35}$ is a strong evidence that water is not to be looked as a mixture of a two local structures. Rather, the presence of two correlations scales separated by $R_{c}$, naturally suggests the picture for liquid water based on correlations, rather than that of clusters based on an instantaneous snapshot vision. In this picture, water is a molecular liquid which has very specific correlations, that I would call "correlons", each of size $2 R_{c}$, which would correspond to the 3PS. Such entities are not necessarily tetrahedrally ordered, as in an instantaneous view would naively suggest, but averages of local order that appear at the density correlation level. Such entities would be ideal between them, or only weakly interact between themselves, which would then explain the strong damping of correlations beyond $R_{c}$. As such, they would freely overlap, which means that there is no particular center for such a correlon, as opposed to a specific cluster. This would explain why specific clusters were never observed, or why there is no strong evidence for a 2-water structure as it is usually pictured. Peculiar clustering features have been reported by some authors lately, in cold water ${ }^{37}$ as well in supercooled water, ${ }^{36}$ and these could have 
a relation with the 3PS reported here. In any case, the analysis shows that water in ambient conditions, despite being a disordered liquid, hides considerable amount of order that extends quite far from a single molecule, and which make it an unusual liquid.

\section{Acknowledgments}

The author thanks Gren Patey for critical reading of the manuscript and enlightening discussions.

\section{References}

[1] W. K. Roentgen, Ann. Phys. U. Chim. (Wied) 45 (1892) 91-97.

[2] L. Pauling, The structure of water, In Hydrogen bonding, Ed. D. Hadzi and H. W. Thompson (Pergamon Press Ltd, London, 1959)

[3] H. S. Frank and A. S. Quist, J. Chem. Phys. 34, 604 (1961)

[4] H. E. Stanley et al. J. Phys. Cond. Mat. 12, A403 (2000)

[5] H. E. Stanley, S. V. Buldyrev, M. Canpolat, O. Mishima, M. R. Sadr-Lahijany, A. Scala and F. W. Starr , PCCP 2, 1551 (2000).

[6] P.G. Debenedetti Nature, 392, 127, (1998).

[7] A. K. Soper, Chem. Phys. 258,121 (2000)

[8] T. Head-Gordon and H. Hura, Chem. Rev. 102, 2651 (2002)

[9] H. J. Bakker, Chem. Rev. 108, 1456 (2008).

[10] M. -C. Bellissent-Funel, Europhys. Lett. 42, 161 (1998)

[11] B. Guillot, J. Mol. Liq. 101, 219 (2002).

[12] E. A. Jagla, Phys. Rev. E58, 1478 (1998)

[13] G. Franzese, J. Mol. Liq. 136, 267 (2007) 
[14] A. B. de Oliveira, P. A. Netz and M. C. Barbosa, Eur. Phys. J. B64, 481 (2008)

[15] C. Huang et al., Proc. Nat. Acad. Sci. 106, 15214 (2009)

[16] C. Huang et al., Proc. Nat. Acad. Sci. 107, E45 (2010)

[17] N. I. Clark, G. L. Hura, J. Teixeira, A. K. Soper and T. HeadGordon, Proc. Nat. Acad. Sci. 106, 15214 (2009)

[18] A. K. Soper, J. Teixeira and T. Head-Gordon, Proc. Nat. Acad. Sci. 107, E44 (2010)

[19] J.P. Hansen and I.R. McDonald, Theory of Simple Liquids (Academic, London, 1986).

[20] J. C. Berendsen, J.P.M. Postma, W.F. Von Gusteren and J. Hermans, in Intermolecular Forces, edited by B. Pullman (Reidel, Dortrecht, 1981).

[21] W.L. Jorgensen, J. Chandrasekhar, J.D. Madura, R.W. Impey and M.L. Klein, J. Chem. Phys. 79, 926 (1983).

[22] M.W. Mahoney and W. L. Jorgensen, J. Chem. Phys. 112, 8910 (2000).

[23] A. Perera, A. Rispe, L. Zoranić, R. Mazighi and F. Sokolić. To appear in Molecular Physics (2009).

[24] W. L. Jorgensen, J. Phys. Chem. 90, 1276 (1986).

[25] W. L. Jorgensen, J. M. Briggs and M. L. Contreras, J. Phys. Chem., 94, 1683 (1990).

[14] J. M. Cordeiro, Int. J. Quantum Chem. 65, 709 (1997).

[26] A. Perera and F. Sokolić, J. Chem. Phys. 121, 11272 (2004).

[27] L. Bosio, J. Teixeira and M.-C. Bellissent-Funel, Phys. Rev. A39, 6612 (1989).

[28] A.B. Bhatia, D. E. Thornton, Phys. Rev. B2, 3004 (1970).

[29] L. Bosio, J. Teixera and H. E. Stanley, Phys. Rev. Lett. 46, 597 (1981). 
[30] H. Tanaka, Phys. Rev. Lett. 80,5750 (1998).

[31] Y. Koga, J. Phys. Chem. 100, 5172 (1996).

[32] D. T. Bowron, J. L. Finney, A. Hallbrucker, I. Kohl, T. Loerting, E. Mayer and A. K. soper, J. Chem. Phys. 125, 194502 (2007)

[33] M. Teubner and R. Strey, J. Chem. Phys. 87, 3195 (1987).

[34] A. Perera, F. Sokolic and L. Zoranic, Phys. Rev. E75, 060502(R) (2007).

[35] L. Zoranic, F. Sokolic and A. Perera, J. Chem. Phys. 127, $024502(2007)$.

[36] J. E. Errington, P. G. Debenedetti and S. Torquato, Phys. Rev. Lett. 89, 215503 (2002)

[37] Ph. Wernet et al., Science 104, 995 (2004) 


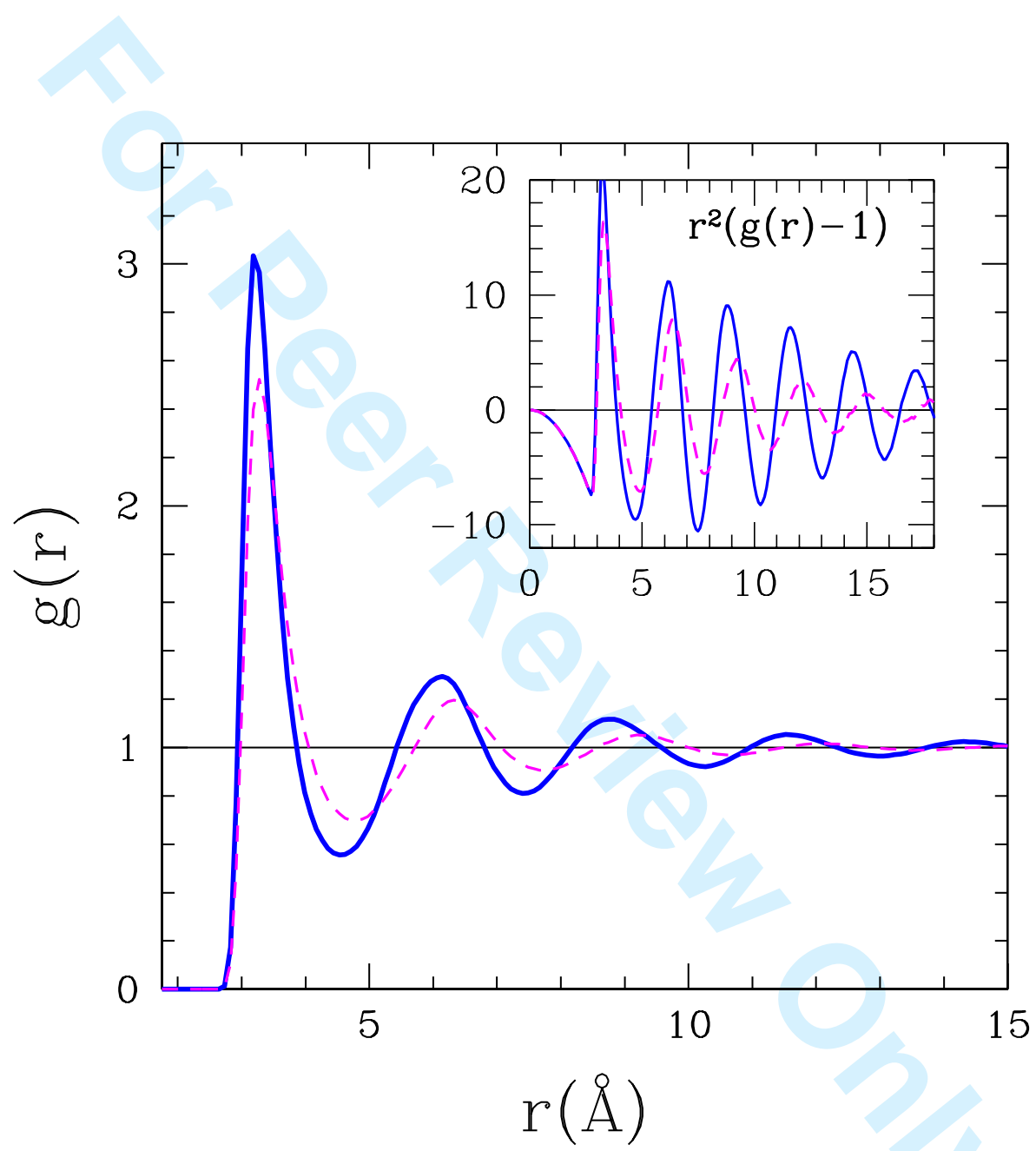

Figure 1: Typical liquid state radial distribution function for reduced densities $\rho^{*} \approx 1$ (full line) and $\rho^{*}=0.73$ (dotted). The inset shows the integrand (note the difference in vertical scale in the inset 


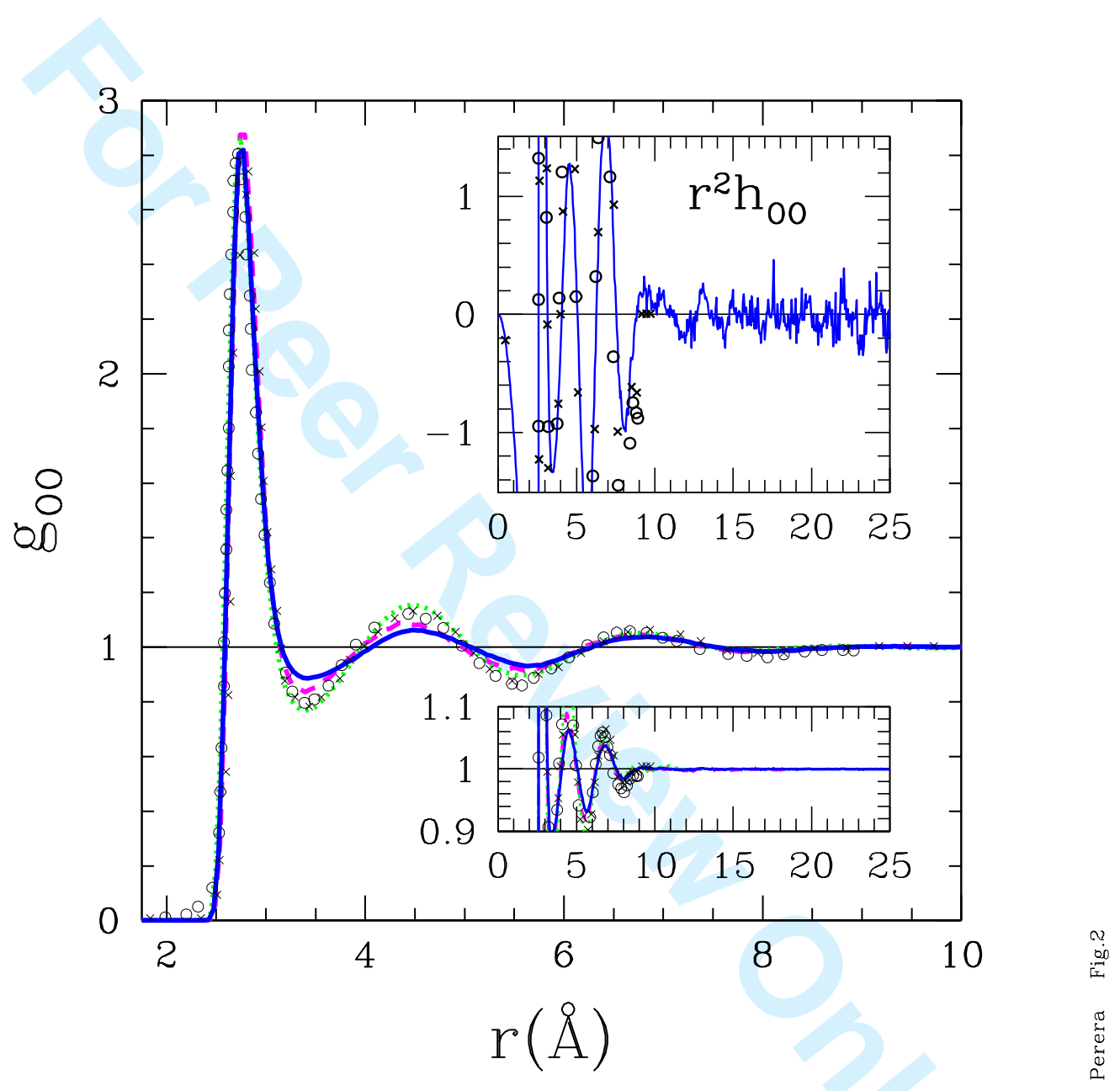

Figure 2: The oxygen-oxygen (OO) RDF for the three water models: SPC/E(full line), TIP4P(dashed) and TIP5P(dotted), experimental data from $\operatorname{Ref}^{7}$ crosses and $\operatorname{Ref}^{8}$ (open dots). The lower inset is a close up of the long range correlations. The upper inset shows the integrand of the compressibility (see text) for the SPC/E mode 


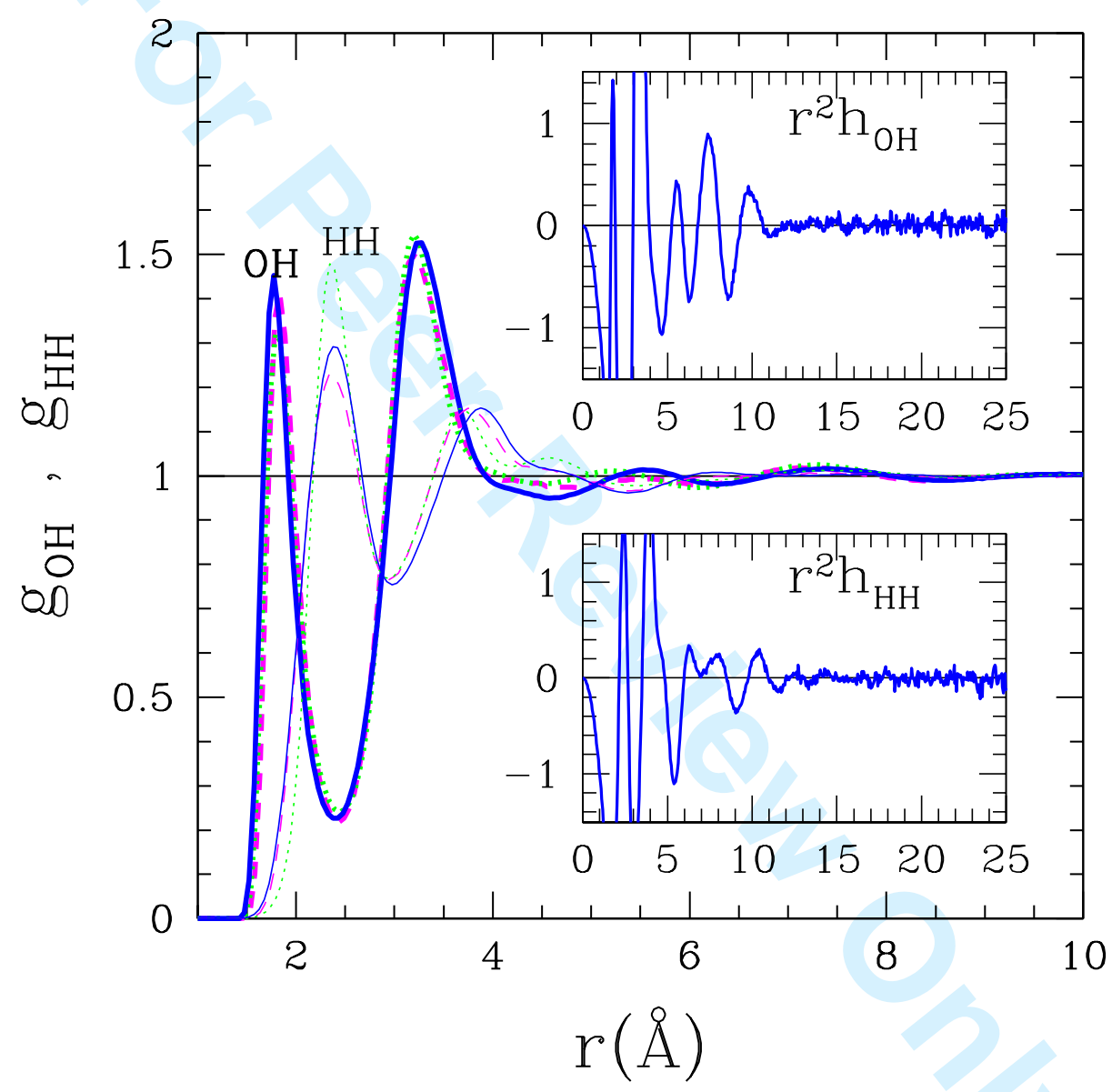

Figure 3: The oxygen-hydrogen and hydrogen-hydrogen radial distribution functions for the three water models: same line code as in Fig.1. The upper inset is a close up of the $\mathrm{OH}$ integrand and the lower inset for the $\mathrm{HH}$ correlations, both for the SPC/E model 


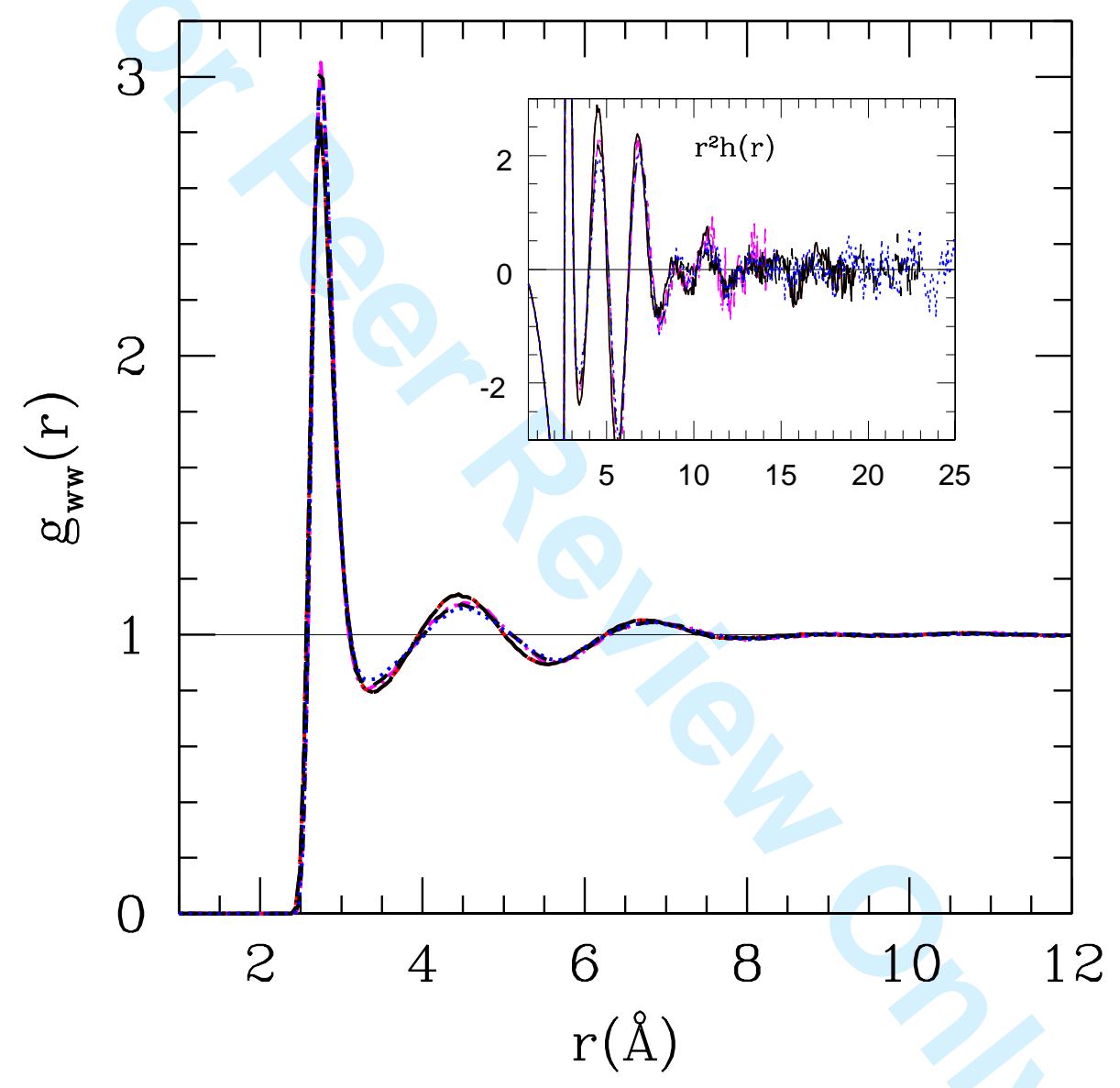

Figure 4: Test of the model and size dependence of the features: full line TIP5P $(\mathrm{N}=2048)$, dash-dotted TIP4P $(\mathrm{N}=2048)$, dashed SPC $/ \mathrm{E}(\mathrm{N}=864)$, big dashes $\mathrm{SPC} / \mathrm{E}=4000)$, dotted $\mathrm{SPC} / \mathrm{E}(\mathrm{N}=10976)$. 


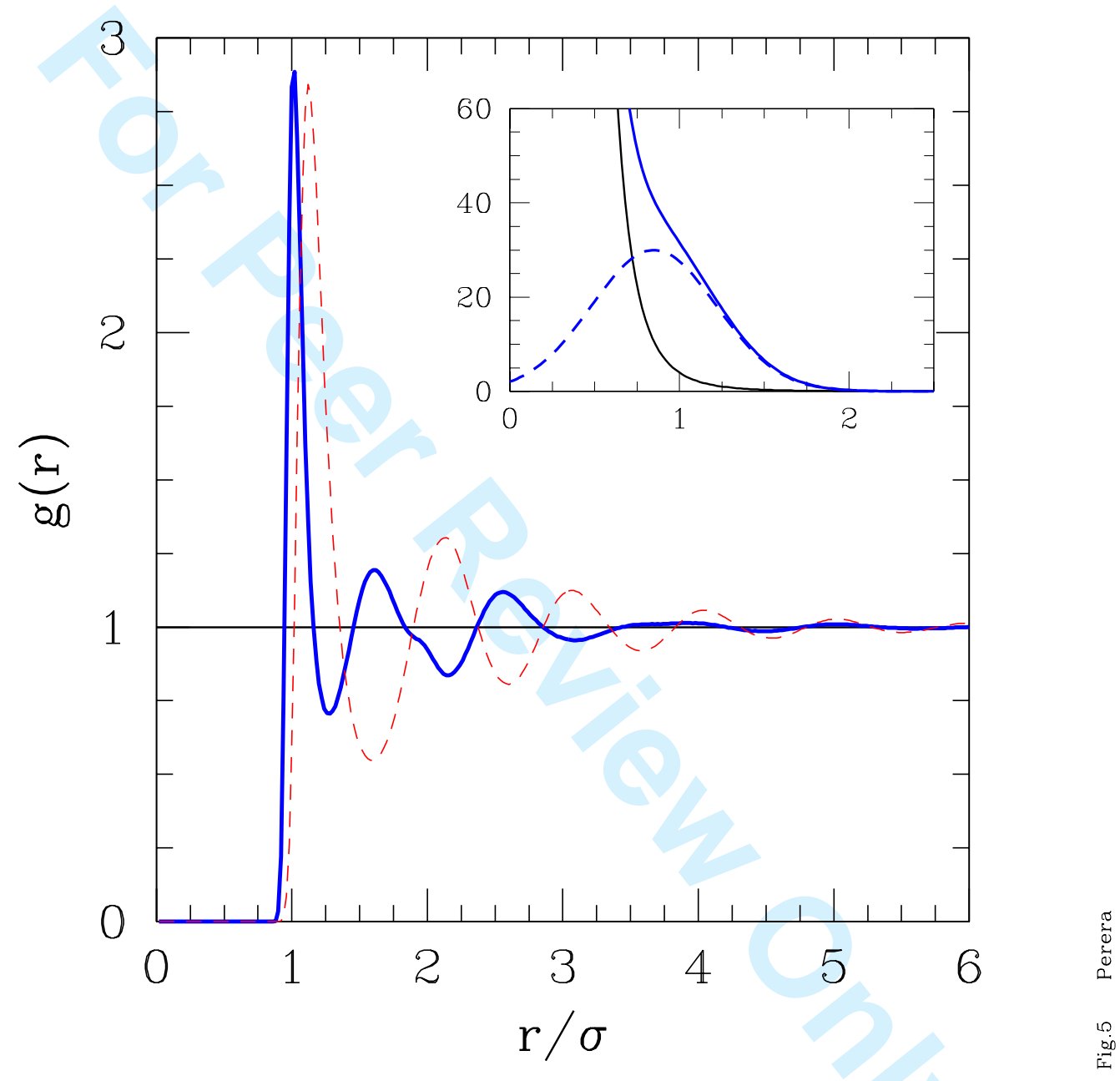

Figure 5: The RDF for the core-softened interaction. Full lines MC simulation results. The dashed curve is the MC RDF for soft repulsion alone. Inset shows the interaction: thinner full line $1 / r^{12}$, dashes for the Gaussian repulsion and thicker full line for core-softened interaction. 


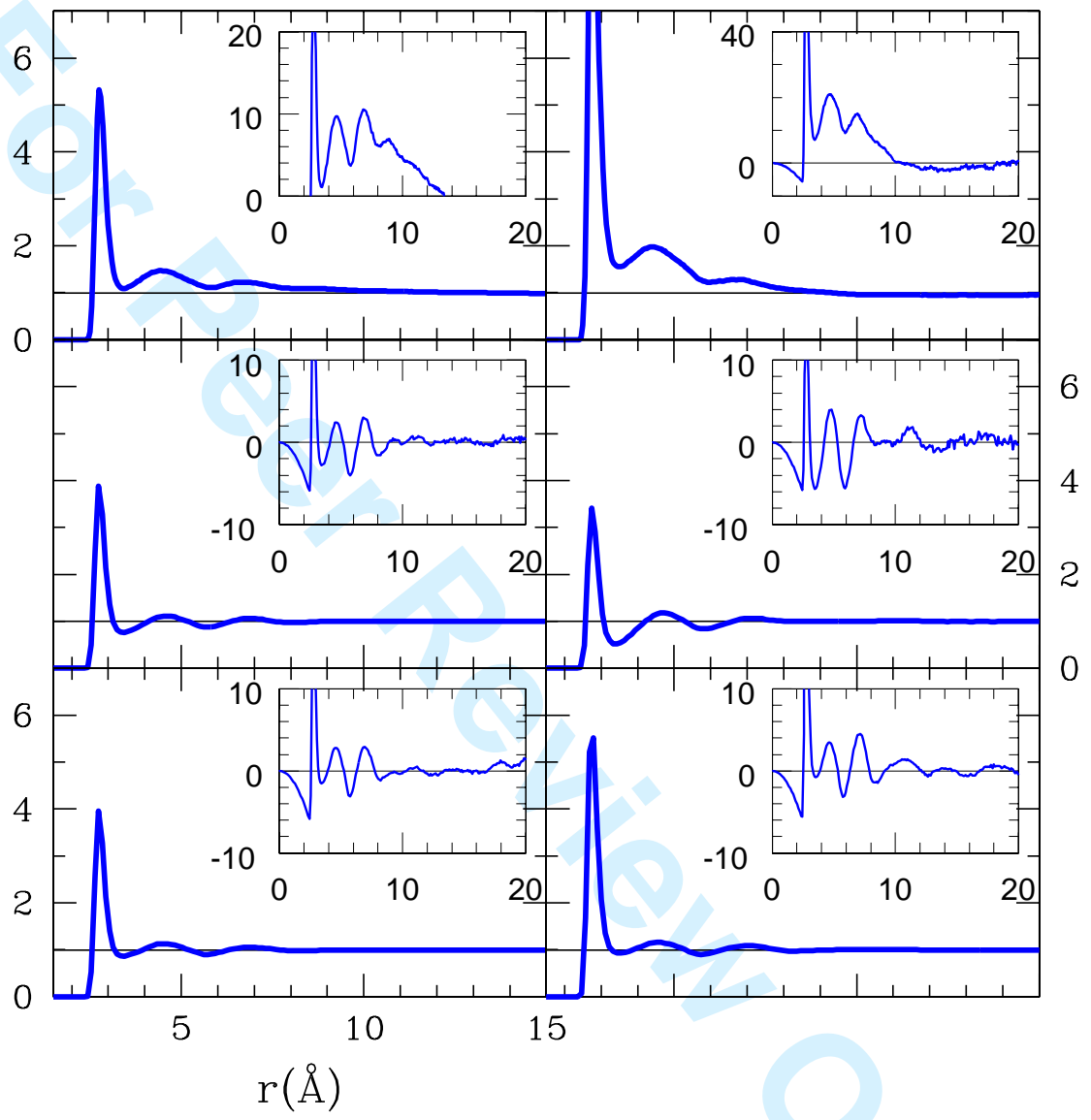

Figure 6: Behaviour of the "3-peaks structure" of the water-water RDFs, for three different binary aqueous mixtures, with three different organic solvents: acetone (the two upper panels), methanol (middle panels) and formamide (lower panels). Left panel is $20 \%$ organic solvent content and right panel for $50 \%$ content. The insets show the integrands. The vertical and horizontal scales are kept the same in all 6 main panels. 


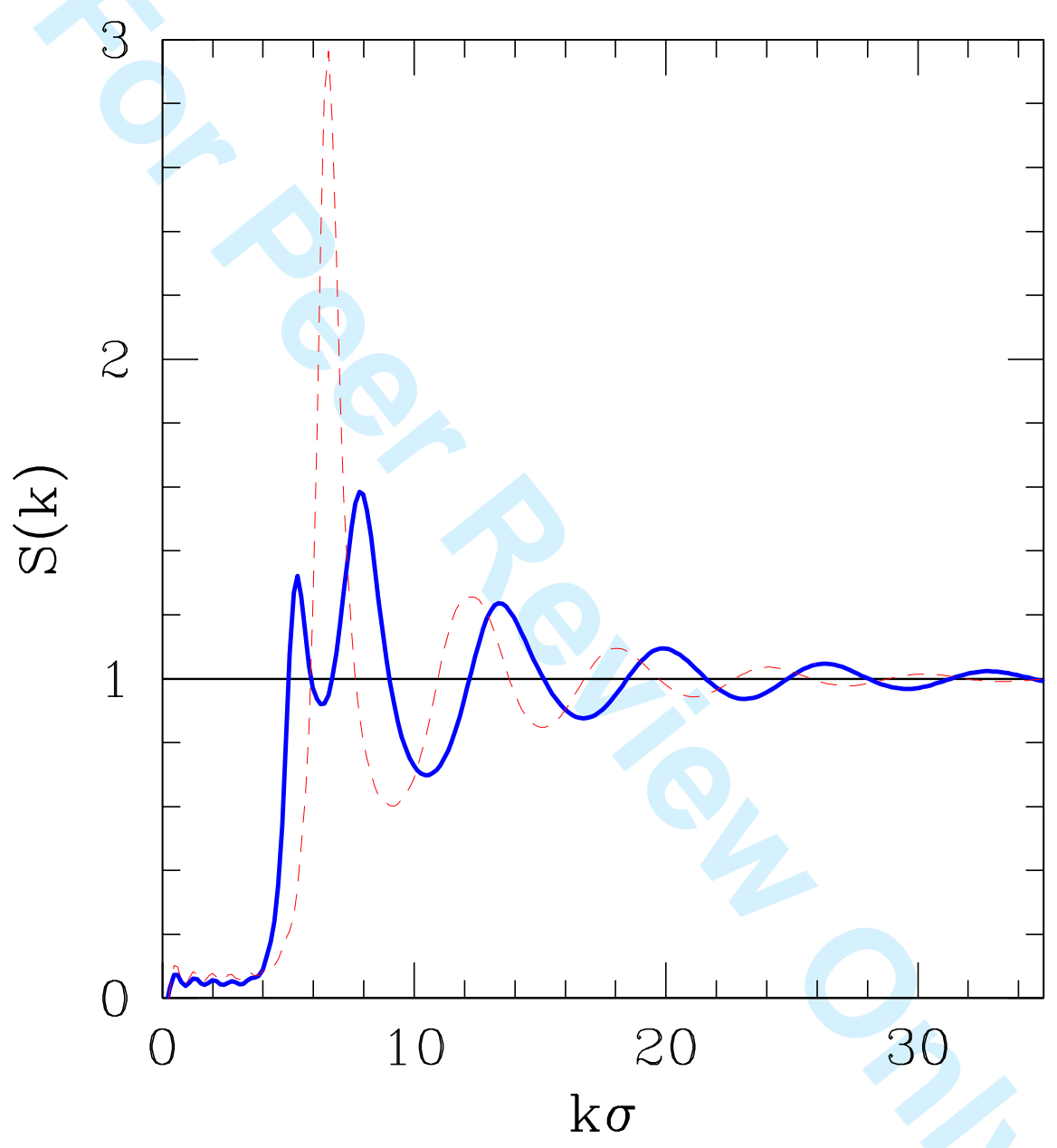

Figure 7: Structure factors corresponding to the RDFs of the core-softened model in Fig.5, with same line conventions 


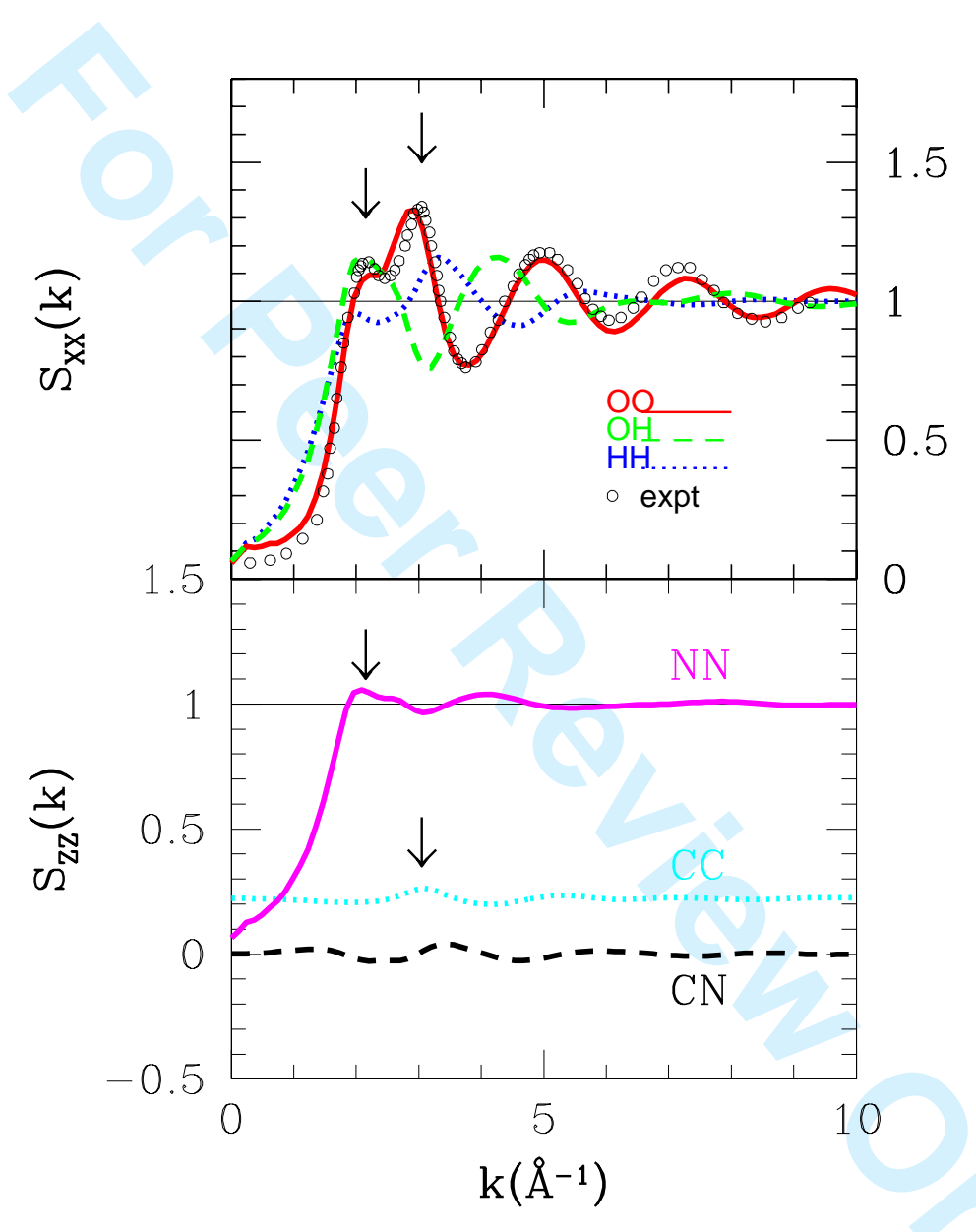

Figure 8: Top panel: the three site-site SFs for the SPC/E water model(OO (full line), $\mathrm{OH}$ (dashed) and $\mathrm{HH}$ (dotted)), with the experimental SF from $\operatorname{Ref}^{8}$ (open dots). The arrows point at the two split-peaks. Bottom panel: the Bhatia-Thornton SFs discussed in the text. The arrows point at the same peak positions as in the upper panel. 